medgen $2016 \cdot 28: 245-250$

DOI 10.1007/s11825-016-0090-6

Online publiziert: 1. September 2016

(c) Der/die Autor(en) 2016. Dieser Artikel ist

eine Open-Access-Publikation.

CrossMark

\section{Einleitung}

Zellen von Tumoren weisen in der Regel eine Vielzahl an somatischen Aberrationen in ihren jeweiligen Tumorgenomen auf. Dazu gehören Basensubstitutionen, Insertionen oder Deletionen kleinerer DNA-Abschnitte (Indels), Kopienzahlabweichungen größerer chromosomaler Abschnitte (engl.: somatic copy number alterations [SCNAs]), strukturelle Umbauten, wie beispielsweise Translokationen oder Insertionen und epigenetische Veränderungen, die die Chromatinstruktur und Genexpression verändern $[1,2]$. Die detaillierte Charakterisierung dieser somatischen Veränderungen in Tumorgenomen spielt in der klinischen Onkologie zunehmend eine bedeutende Rolle, weil sie das Potenzial hat, prognostische und prädiktive Biomarker zu identifizieren. Die Untersuchung von Primärtumoren oder Metastasen hat aber mehrere systemimmanente Limitationen, weil sie immer nur den Zustand des Tumorgenoms zum Zeitpunkt der Gewebeentnahme reflektieren kann und aufgrund der Tumorheterogenität das untersuchte Areal des Tumors oder der Metastase nicht repräsentativ sein muss. Da es sich jeweils um invasive Untersuchungsverfahren handelt, sind die Gewebeproben je nach Lokalisation oft nur schwer zu entnehmen und ihre Entnahme kann nicht unbegrenzt zu verschiedenen Zeitpunkten wiederholt werden. Da Tumorgenome eine hohe Instabilität aufweisen und sich unter Selektionsdrücken wie verabreichten Therapien ändern können, ist eine Überwa-

\author{
Michael R. Speicher ${ }^{1} \cdot$ Armin Gerger $^{2} \cdot$ Gerald Hoefler $^{3}$ \\ ${ }^{1}$ Institut für Humangenetik, Medizinische Universität Graz, Graz, Österreich \\ ${ }^{2}$ Universitätsklinik für Innere Medizin, Klinische Abteilung für Onkologie, Medizinische Universität Graz \\ Graz, Österreich \\ ${ }^{3}$ Institut für Pathologie, Medizinische Universität Graz, Graz, Österreich
}

\title{
Liquid Biopsies
}

\section{Eine interdisziplinäre Herausforderung für Humangenetik, Onkologie und Pathologie}

chung des Tumorgenoms auf mögliche neue Aberrationen eine wesentliche Voraussetzung für den Einsatz zielgerichteter Therapien, die auf Charakteristika des Tumorgenoms beruhen $[1,2]$.

Bestimmte Komponenten von Tumoren lassen sich in der Blutzirkulation von Krebspatienten nachweisen. Dabei handelt es sich um zirkulierende Tumorzellen (engl.: circulating tumor cells [CTCs]), zirkulierende Tumor-DNA (engl.: circulating tumor DNA [ctDNA]) oder auch um Exosomen [3]. CTCs sind Zellen, die vom Primärtumor und/oder Metastasen in die Blutzirkulation gelangen, aber dort selbst bei metastasierten Krebserkrankungen in der Regel nur in sehr geringer Zahl (1 CTC pro $1 \times 10^{9}$ normale Blutzellen) im Blut nachgewiesen werden können, sodass ihre Identifizierung und Isolation für nachfolgende Analysen ein spezielles Instrumentarium benötigt [4]. ctDNA gelangt nach heutigem Kenntnisstand hauptsächlich von apoptotischen Zellen nach enzymatischem Verdau in die Blutzirkulation. Selbst bei metastasierten Patienten kann der ctDNA Gehalt sehr unterschiedlich sein [5]. Exosomen sind Vesikel, die unterschiedliche Komponenten von Tumorzellen, wie Nukleinsäuren oder Proteine beinhalten können. Neuste Techniken für ihre Isolation und Analyse haben sie zu einem vielversprechenden Biomarker werden lassen [6]. In den vergangenen Jahren wurden zahlreiche Techniken und Verfahren entwickelt, um insbesondere CTCs und ctDNA diagnostischen Zwecken zugänglich zu machen. Dies resultierte in einer enormen Anzahl an Publikationen zahlreicher Arbeitsgruppen, die zeigten, dass CTCs oder ctDNA verwendet werden können, (1) um Krankheitsverläufe bei Krebspatienten zu überwachen, insbesondere auch zur Erfassung des Remissionsstatus (minimal residual disease [MRD]) durch hochsensitive Verfahren, (2) zur Identifizierung von neu auftretenden Resistenzmechanismen bei Patienten unter gegebenen zielgerichteten Therapien, (3) zur Analyse der Tumorheterogenität und (4) allgemein zur Identifizierung neuer tumorspezifischer Veränderungen, die im Krankheitsverlauf auftreten können (für aktuelle Übersichtsarbeiten siehe [5, 7-11]). Auf der Basis dieser vielversprechenden Ansätze könnten sich Blutabnahmen mit nachfolgenden entsprechenden Analysen zu einer Alternative zu Gewebebiopsien entwickeln, weshalb sie auch als „flüssige Biopsien“ (engl.: liquid biopsies) bezeichnet werden. Die offensichtlichen Vorteile der Liquid Biopsies liegen darin, dass sie nicht invasiv sind (oder als Blutabnahme „minimal-invasiv“) und deshalb ohne zusätzliche Belastung für Patientinnen und Patienten auch leicht zu mehreren Zeitpunkten wiederholt werden können. Den Liquid Biopsies wird ein immenses Potenzial eingeräumt, die sogenannte personalisierte Medizin und insbesondere den Einsatz zielgerichteter Therapien revolutionieren zu können, sodass allgemein davon ausgegangen wird, dass diese Technologien zeitnah eine breite Anwendung finden werden. Im Nachfolgenden fassen wir zusammen, welche Voraussetzungen für den klinischen Routineeinsatz der Liquid Biopsies erfüllt werden müssen, wenn umfassende 
Aussagen über genetische Veränderungen getroffen werden sollen, und welche Fachdisziplinen für ihre Umsetzung einbezogen werden müssen.

\section{Liquid Biopsies: Schon bereit für den klinischen Routineeinsatz?}

Eine zentrale Frage ist, inwieweit die Liquid Biopsies zum jetzigen Zeitpunkt bereits für den klinischen Routinealltag eingesetzt werden können. Trotz des unbestritten großen Potenzials der Liquid Biopsies und der diesbezüglich teilweise spektakulären publizierten Resultate (siehe vorgenannte Übersichtsarbeiten), ist es zurzeit für den Routineeinsatz außerhalb von definierten Studien noch ein weiter Weg. Ein diesbezüglich im letzten Jahr abgehaltener Workshop (Policy Issues in the Development and Adoption of Biomarkers for Molecularly Targeted Cancer Therapies; http://www.nap. edu/catalog.php?record_id=21692) hat bestehende Defizite detailliert aufgelistet [12]. Diese Defizite fangen bereits damit an, dass zurzeit einheitliche Verfahrensanweisungen (SOPs) für eine standardisierte Probengewinnung, Verarbeitung und Lagerung fehlen. Bei der Befunderstellung gibt es in vielen Bereichen keine ausreichenden Standards für Untersuchungsverfahren und Ergebnisse und welche minimalen Berichtanforderungen ein Befund enthalten soll. Es fehlen etablierte Richtlinien, welche Varianten in einem Tumorgenom als "therapierbar" einzustufen sind und an welchen Quellen man sich für diese Einordnung orientieren soll. Von wenigen Ausnahmen abgesehen (z. B. ERBB2/HER2 Amplifikation und Therapie mittels Antikörper gegen diesen epidermalen Wachstumsfaktorrezeptor wie Trastuzumab) fehlen Regularien, um mögliche therapeutische Konsequenzen aufgrund spezifischer somatischer genetischer Testergebnisse festzulegen. Dies könnte sich durch Initiativen wie das Actionable Genome Consortium (AGC) verbessern [13], welches versucht, Behandlungsstrategien aufgrund von next generation sequencing (NGS)-Daten zu erstellen. Es fehlen auch globale Bestimmungen, die länderübergreifend die Verwendung von Biomarkertests regu- lieren und harmonisieren [12]. All diese Defizite treffen sowohl für prädiktive als auch für prognostische Biomarker zu.

Die Bezeichnung "Liquid Biopsy" beschreibt keinen einheitlichen Test, sondern bezieht sich auf ein Sammelsurium unterschiedlicher Techniken, die auf CTCs oder ctDNA angewendet werden können. Dabei kann es sich um sehr fokussierte Verfahren handeln, die spezifisch nur eine Mutation in einem Gen analysieren (z. B. aktivierende RAS Mutationen bei Patienten mit kolorektalen Karzinomen unter anti-EGFR Therapie) oder auch um extensive Ansätze wie whole-exome oder whole-genome Analysen, die auf NGS beruhen. Gerade die letztgenannten Untersuchungen haben das Potenzial, Daten und Informationen $\mathrm{zu}$ generieren, die weit über eine Erhebung rein somatischer, tumorspezifischer Varianten hinausgehen. Je mehr Gene in eine Untersuchung inkludiert werden, desto größer wird die Wahrscheinlichkeit, dass auch Varianten nachgewiesen werden, die nicht somatisch, sondern in der Keimbahn vorhanden sind. Der Nachweis einer solchen Variante, z. B. in einem Tumorsyndrom prädisponierenden Gen, hätte aber erhebliche Konsequenzen, die weit über die ursprüngliche Fragestellung hinausgehen und würde unmittelbar eine Beratungsnotwendigkeit weiterer Familienmitglieder nach sich ziehen.

Deshalb müssen bei Liquid Biopsies Fragen wie Einverständniserklärung, Umfang der Aufklärung und Umgang mit Zusatzbefunden geklärt werden. Es muss sichergestellt werden, dass Ergebnisse aus Liquid Biopsies einheitlich interpretiert werden. Dies trifft nicht nur für potenziell prädiktive und prognostische Biomarker, sondern gegebenenfalls auch für Varianten der Keimbahn zu [12].

$\mathrm{Zu}$ diesem Zweck wurde in Europa das Cancer-ID Consortium konstituiert (http://www.cancer-id.eu/), das aus 33 akademischen und industriellen Partnern aus 13 Ländern besteht und von der Innovative Medicines Initiative (IMI) unterstützt wird. Hauptziel dieses fünfjährigen Projektes, welches im Januar 2015 gestartet wurde, ist die Etablierung von SOPs für den Einsatz von
Liquid Biopsies und die klinische Validierung von auf Bluttests basierenden Biomarkern. Dieses ambitionierte Projekt würde einen Vergleich von Analysen, die an verschiedenen Standorten erhoben werden, deutlich vereinfachen und wäre eine wesentliche Voraussetzung für multizentrische Studien, die dringend notwendig sind, um mit großen Fallzahlen die Liquid Biopsies klinisch zu validieren. Schließlich stehen zurzeit die Bestätigung der analytischen Validität (Genauigkeit, mit der ein Test das spezifische Analyt, für das er entwickelt wurde, auch tatsächlich entdeckt), der klinischen Validität (Maß für die Genauigkeit eines Tests für eine spezifische klinische Verwendung, z.B. Auswahl einer zielgerichteten Therapie in einer ausgewählten Patientengruppe) und des klinischen Nutzens (clinical utility: Kann der Test tatsächlich für klinische Entscheidungen eingesetzt werden und inwieweit profitieren betroffene Patienten?) von Liquid Biopsies noch aus. Gerade der letzte Punkt wird dadurch erschwert, dass es keine Standards gibt, um die Effektivität von diagnostischen Daten zu vergleichen.

Die Etablierung von Liquid Biopsies für klinische Anwendungen würde auch erheblich von dem Aufbau von Datenbanken profitieren, die den Austausch, Vergleich und die Interpretation von Informationen erleichtern, was natürlich wieder entsprechende Richtlinien und gegebenenfalls Gesetzesvorgaben verlangen würde, die ethische und rechtliche Belange eines solchen Datenaustausches und einer systematischen Datenbanksammlung berücksichtigen [12].

Wenn Liquid Biopsies für Therapieentscheidungen herangezogen werden sollen, müssen Laborqualitätsstandards entwickelt und erfüllt werden. Die Anforderungen für diejenigen Liquid Biopsies, bei denen es sich vornehmlich um genetische Analysen handelt, würden sich diesbezüglich nicht von bereits etablierten, internationalen Standards für humangenetische Untersuchungen unterscheiden, sodass sie nur in entsprechend akkreditierten Labors durchgeführt werden sollten. Eine wichtige Herausforderung wird es sein, die Analysen in einem kurzen, angemessenen 
Zeitraum zu erstellen, da die Betroffenen und ihre behandelnden Ärztinnen und Ärzte die Ergebnisse zeitnah zur Festlegung der weiteren Vorgehensweise benötigen. Zusätzlich müssen auch bei Liquid Biopsies Ringversuche zur Qualitätssicherung etabliert werden.

Zusammenfassend haben wir keinen Zweifel, dass sich die Liquid Biopsies trotz der vorgenannten Einschränkungen zu einem wichtigen diagnostischen Zweig bei der Betreuung von Krebspatienten entwickeln werden. Gleichzeitig müssen aber vor einem klinischen Routineeinsatz noch viele offene Fragen und ungelöste Probleme geklärt werden. Aus diesem Grund sollte derzeit der Einsatz der Liquid Biopsies auf definierte Studien beschränkt bleiben.

\section{Liquid Biopsy: Stakeholders und involvierte Fachbereiche}

Die wichtigsten Stakeholder bei Liquid Biopsies sind natürlich die Patientinnen und Patienten, die sich von diesen verbesserte Informationen über ihren Krankheitsverlauf und insbesondere über Therapieoptionen erhoffen. Da die Liquid Biopsies die praktizierte Medizin in zahlreichen Gebieten - auch über die Onkologie hinaus - verändern werden, ist es unser Auftrag, das Potenzial dieser vielversprechenden Techniken systematisch auszutesten und daraufhinzuwirken, dass diese - nach Validierung - in den klinischen Alltag eingeführt werden.

Die zahlreichen Aufgaben, die vor dem klinischen Routineeinsatz der Liquid Biopsies erledigt werden müssen, können aber nicht von einer einzelnen Gruppierung allein gestemmt werden, vielmehr erfordern sie einen multidisziplinären Ansatz. Dies stellt insbesondere die Humangenetik vor neue Herausforderungen: Im Rahmen einer Pilotstudie zur „personalisierten Onkologie durch integrative Hochdurchsatz-Sequenzierung" wurde von Arul Chinnaiyans Arbeitsgruppe bereits 2011 der Ablauf der einzelnen Schritte für die Betreuung von Patientinnen und Patienten im Zeitalter der Hochdurchsatz-Sequenzierung dargestellt (• Abb. 1; [14]).

Aufgrund der zunehmenden Sequenzieraktivitäten wird sich das Procedere

medgen 2016 28:245-250 DOI 10.1007/s11825-016-0090-6

(c) Der/die Autor(en) 2016. Dieser Artikel ist eine Open-Access-Publikation.

\section{R. Speicher · A. Gerger · G. Hoefler}

\section{Liquid Biopsies. Eine interdisziplinäre Herausforderung für Humangenetik, Onkologie und Pathologie}

\section{Zusammenfassung}

"Liquid Biopsy" bezieht sich in der Onkologie auf die Untersuchung von zirkulierenden Tumorzellen (engl.: circulating tumor cells [CTCs]) oder zirkulierender Tumor-DNA (engl:: circulating tumor DNA [ctDNA]), um nicht invasiv aus dem peripheren Blut Charakteristika über das Tumorgenom von Krebspatienten zu erfassen. Den Liquid Biopsies wird ein immenses Potenzial für zukünftige Entwicklungen in der personalisierten Medizin und dem Einsatz zielgerichteter Therapien eingeräumt. In dieser Übersichtsarbeit zeigen wir, dass aber noch viele offene Fragen angegangen werden müssen, bevor Liquid Biopsies für die klinische Routine eingesetzt werden können. Eine besondere
Herausforderung liegt darin, dass Ergebnisse je nach gewähltem Untersuchungsverfahren eine Tragweite haben können, die weit über die ursprünglich beabsichtigte Untersuchung des Tumorgenoms hinausgeht. All diese Fragen können nur in einem interdisziplinären Ansatz unter Beteiligung der Humangenetik, Onkologie, Pathologie, Bioinformatik und Bioethik angegangen und beantwortet werden.

\section{Schlüsselwörter}

ctDNA-Analysen - Tumordiagnostik .

Zirkulierende Tumorzellen . Personalisierte Medizin · NGS-Analysen von Tumorgenomen

\section{Liquid biopsies. A multidisciplinary challenge for human geneticists, oncologists, and pathologists}

\section{Abstract}

In oncology, "liquid biopsy" refers to the analysis of circulating tumor cells (CTCS) or of circulating tumor DNA (ctDNA), to establish non-invasively from the peripheral blood the characteristics of a tumor genome in cancer patients. Liquid biopsies have tremendous potential for future developments in personalized medicine and for the application of targeted therapies. In this review we show that many unresolved issues need to be addressed before liquid biopsies can be routinely used. A particular challenge is the fact that, depending on the analysis performed, the results can have implications reaching far beyond the analysis of the tumor genome originally intended. All of these issues can best be addressed in a multidisciplinary setting with human geneticists, oncologists, pathologists, bioinformaticians and bioethicists participating.

\section{Keywords}

Circulating tumor DNA (ctDNA) analysis . Tumor diagnosis - Circulating tumor cells . Personalized medicine $\cdot$ Next generation sequencing (NGS) of tumor genomes für die benötigte Aufklärung der Patientinnen und Patienten ändern und sich die Notwendigkeit für ein humangenetisches Beratungsgespräch schon oft zum Zeitpunkt der Erstdiagnose ergeben, insbesondere dann, wenn das Tumorgenom nicht nur auf wenige etablierte prädiktive Varianten (z. B. KRAS/NRAS beim Kolonkarzinom), sondern umfassend untersucht werden soll. Der Beratungsbedarf ergibt sich allein daraus, dass wholeexome oder whole-genome sequencing Analysen von Tumorgenomen ein sehr hohes Potenzial haben, nicht nur somatische, sondern auch Keimbahnvarianten von hoher Relevanz zu identifizieren.
Dieses Erstgespräch sollte gegebenenfalls interdisziplinär, zusammen mit Kolleginnen und Kollegen aus der Onkologie und Psychologie stattfinden.

Die Bildung neuer Boards, d. h. multidisziplinärer Sequenzierungs-Tumorboards (bestehend aus Expertinnen und Experten aus den Fachbereichen Onkologie, Humangenetik, Bioinformatik, Pathologie, gegebenenfalls Bioethik) wird unabdingbar werden (• Abb. 2; [14]). Diese Boards haben die Aufgabe, anhand der erhobenen komplexen Datensätze den Tumor molekular zu klassifizieren und etwaige Keimbahnvarianten sowie prognostische und prädiktive Biomarker 


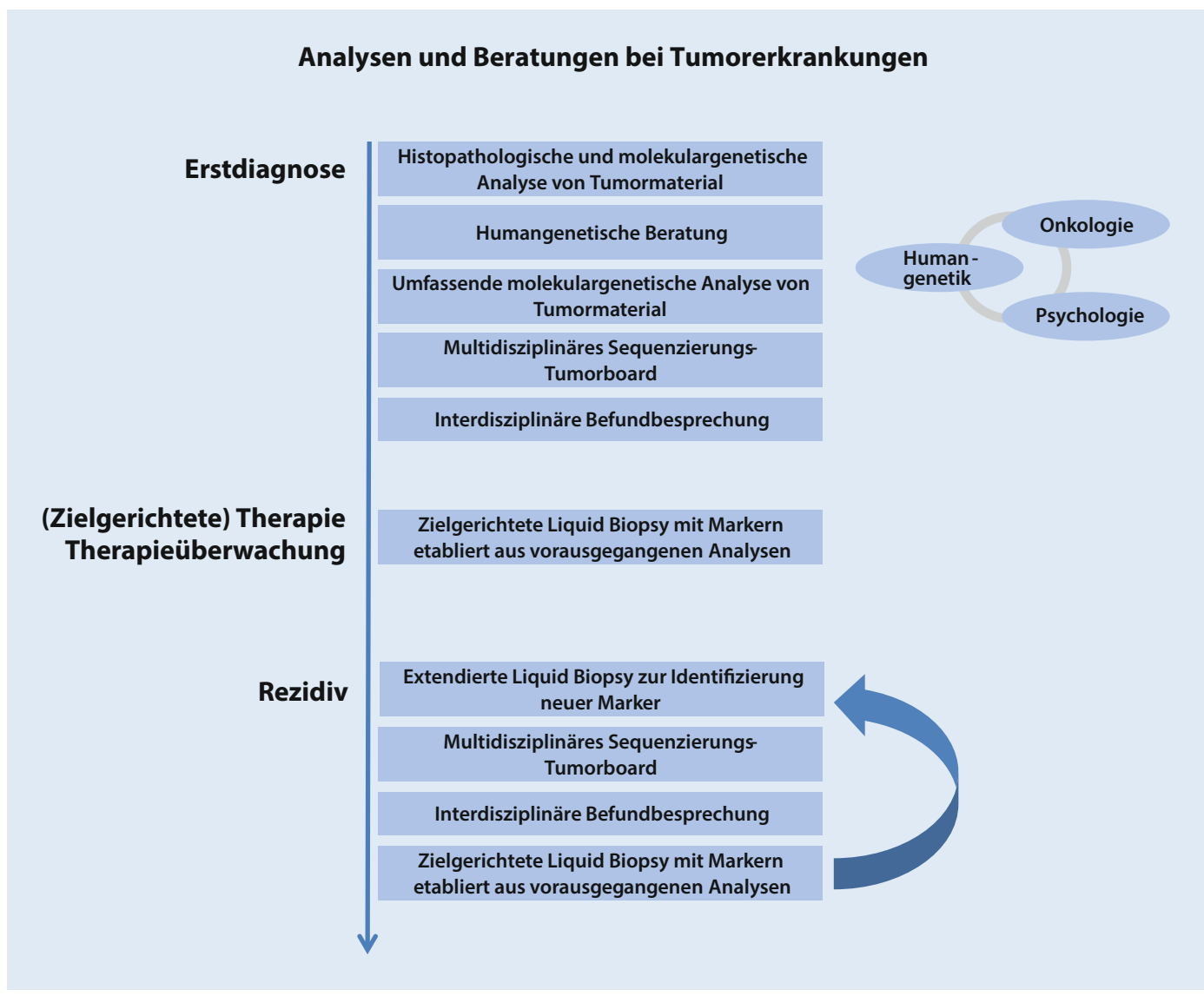

Abb. 1 A Ablauf der Betreuung von Patientinnen und Patienten mit Krebserkrankungen unter Einschluss von Hochdurchsatz-Sequenzierungen des Primärtumors und Liquid Biopsies. Zum Zeitpunkt der Erstdiagnose findet bereits ein erstes humangenetisches Beratungsgespräch, gegebenenfalls multidisziplinär mit Kolleginnen und Kollegen aus der Onkologie und Psychologie, statt. Betroffene werden über geplante Sequenzierungen des Tumorgenoms und möglicherweise der Keimbahn aufgeklärt; Schwerpunkte liegen - neben der obligatorischen Erhebung der Familienanamnese - in der Erläuterung von somatischen und Keimbahnmutationen, möglichen Relevanz von Varianten in der Keimbahn für andere Familienmitglieder und Aufklärung über prädiktive und prognostische Biomarker. Nach Einholung der Einverständniserklärung und nach Operation oder Biopsieentnahme wird das Tumormaterial molekulargenetisch analysiert. Die erhobenen Daten werden von einem multidisziplinären Tumorboard ( $\bullet$ Abb. 2) diskutiert, was idealerweise zu einer optimierten, zielgerichteten Therapie führt, die im Rahmen einer interdisziplinären Befundbesprechung mitgeteilt wird. Nachfolgend wird der Verlauf mittels Liquid Biopsies überwacht, d.h. es wird zielgerichtet die Frequenz spezifischer Marker des Tumorgenoms, die aufgrund der vorausgegangenen Analysen ausgewählt wurden, in der Plasma DNA bestimmt. Sollte zu einem späteren Zeitpunkt ein Progress oder ein Rezidiv diagnostiziert werden, wären wieder erweiterte Liquid Biopsy Analysen notwendig, um beispielsweise zu eruieren, ob sich Resistenzmarker nachweisen lassen oder ob im Rahmen der Tumorprogression neue Veränderungen im Tumorgenom aufgetreten sind. Zu diesen Zeitpunkten ist wieder eine intensive, gegebenenfalls interdisziplinäre Befundmittteilung und Aufklärung über mögliche prognostische und prädiktive Hinweise notwendig. Diese Schritte werden jeweils bei wiederholtem Progress/Rezidiv wiederholt. (Die Abbildung wurde adaptiert von Fig. 1 in [14])

abzuleiten. Die Ergebnisse werden dann im Rahmen einer interdisziplinären Befundbesprechung den Betroffenen vermittelt.

Da komplexe genetische Datensätze erhoben werden, ist die Humangenetik als das Fach mit der umfangreichsten Expertise bzgl. der Interpretation von Varianten des menschlichen Genoms und deren verständlichen Vermittlung an Laien, gefragt. Zusätzlich müssen sich Humangenetiker in das multidis- ziplinäre Sequenzierungs-Tumorboard einbringen (• Abb. 2) und an der Erstellung von Befunden, Empfehlungen und anschließenden Befundmitteilung aktiv mitarbeiten. Die besondere Expertise von Pathologen liegt in der Erfassung und der Interpretation von Veränderungen im Tumorgewebe. Dies inkludiert die histopathologische Untersuchung, die Analyse der Proteinexpression mittels Immunhistochemie, die Untersuchung des Tumorgenoms sowie die Analyse der mRNA-Expression mittels In-situTechniken. Die Beurteilung des Gehaltes an Tumorzellen ist zur korrekten Interpretation von Mutationen (Keimbahnvs. somatischen Mutationen) unerlässlich und stellt einen wichtigen Beitrag im multidisziplinären SequenzierungsTumorboard dar.

Bei der sich anschließenden Therapieüberwachung mittels Liquid Biopsies handelt es sich in der Regel um die zielgerichtete Analyse von somatischen $\mathrm{Mu}$ - 


\section{Multidisziplinäres Sequenzierung-Tumorboard}

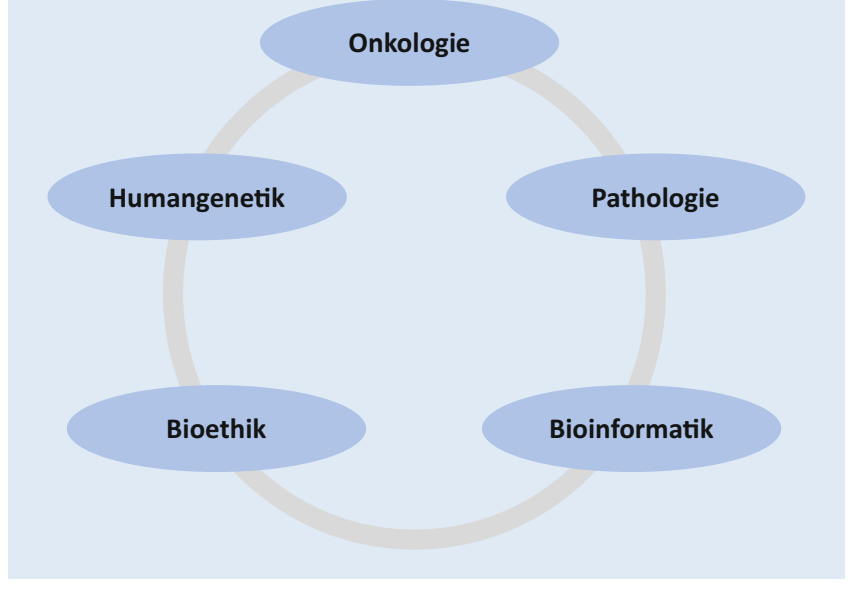

Abb. $2<$ Zusammensetzung eines multidisziplinären Sequenzierung-Tumorboards, das die Expertise aus Onkologie, Humangenetik, Pathologie, Bioethik und Bioinformatik zusammenführt. (Die Abbildungen 1 und 2 wurden adaptiert von Fig. 1 in [14]) tationen des Tumorgenoms, die aus dem Primärtumor diagnostiziert wurden. Sinkende bzw. nicht mehr nachweisbare Marker werden als Therapieansprechen, während steigende Werte als Therapieresistenz oder Rezidiv interpretiert werden. Da es sich bei diesen Analysen also mehr oder weniger „nur“ um die Erfassung steigender oder sinkender Biomarker handelt, die relativ ähnlich $\mathrm{zu}$ anderen Untersuchungen (z. B. bildgebende Verfahren, Tumormarker) sind, besteht hier kein zusätzlicher Beratungsbedarf für die Patienten von humangenetischer Seite.

Im Fall eines Rezidivs oder einer Tumorprogression werden wieder umfassendere Liquid Biopsy Analysen anstehen, weil hier zusätzlich zu den zuvor etablierten Markern nach neuen somatischen Veränderungen gesucht wird (umfassende Mutationsanalyse). Nach unseren eigenen Erfahrungen können diese Analysen im Falle einer Tumorprogression zum Teil ausgiebige, genomweite Veränderungen aufweisen, die nur durch entsprechende umfassende Analysen erkannt werden können [15]. Je nach Ergebnis wird wieder eine umfangreiche, gegebenenfalls interdisziplinäre Befundmittteilung nötig sein (• Abb. 1). Unter bestimmten Bedingungen ist auch eine Analyse von Gewebe aus Metastasen notwendig, um die Ergebnisse entsprechend abzusichern.

Für die Humangenetik ergeben sich mehrere neue Herausforderungen. Falls nicht schon vorhanden, muss eine entsprechende multidisziplinäre Zusammenarbeit mit den genannten Fachdisziplinen etabliert und neue Kapazitäten bereitgestellt werden, um sich von humangenetischer Seite an Boards zu beteiligen und sich in die kontinuierliche Betreuung onkologischer Patientinnen und Patienten mit regelmäßigem Kontakt mit einzubringen, was eine Erweiterung der humangenetischen Beratungsportfolios bedeutet. Bei den Analysen des Primärtumors oder einer Metastase, sei es ein Operationspräparat oder eine Biopsie, handelt es sich um eine gewebsbasierende Diagnostik, sodass diese in Graz am Institut für Pathologie durchgeführt werden. Dies inkludiert die primäre histopathologische Befunderstellung und die Auswahl von Gewebsarealen für nachfolgende Analysen. Da es sich bei der Analyse von ctDNA naturgemäß um keine gewebsbasierende Diagnostik handelt, können diese Analysen auch in humangenetischen Instituten durchgeführt werden. Voraussetzung wäre hier eine interdisziplinäre Zusammenarbeit mit der Pathologie, weil ein Abgleich mit Befunden aus Geweben (entweder Material zum Zeitpunkt der Erstdiagnose oder aus Biopsien während des Verlaufes) erforderlich ist. Es ist unbedingt notwendig, dass die Analysen zeitgerecht (schnell) durchgeführt werden, da wichtige Entscheidungen von diesen abhängen und sie kostengünstig durchgeführt werden müssen. Alle
Analysen, ob aus Gewebe oder Blut, sollten ausschließlich unter etablierten internationalen Qualitätsstandards, wie für humangenetische und pathologische Untersuchungen vorgesehen, in entsprechend akkreditierten Einrichtungen stattfinden.

Falls es an den akademischen Einrichtungen nicht gelingen sollte, den hier skizzierten multidisziplinären Ansatz zu etablieren, wäre auch ein ganz anderes Szenario denkbar: Bereits jetzt gibt es eine zunehmende Anzahl an Firmen, die einen entsprechenden Liquid Biopsy Testservice anbieten (aktuelle Liste von Firmen in [16]). Deshalb könnte Blut auch an kommerzielle Firmen versandt werden und Kliniker könnten nach den Vorgaben in den entsprechenden Befundberichten handeln. In diesem Fall würde die weitere Entwicklungsund Forschungsarbeit, die unbedingt notwendig ist, nicht mehr von akademischen Institutionen beeinflusst, sondern anderen überlassen werden. Der klinisch-akademische Bereich würde dann in diesem zukunftsträchtigen Feld auf eine Zuschauer- oder Konsumentenrolle reduziert werden und eine aktive Entwicklungsarbeit einstellen. Dies hätte erhebliche Nachteile für alle Stakeholder, sodass vertrauensvolle multidisziplinäre Kooperationen alternativlos sind.

\section{Zusammenfassung und Ausblick}

Die erfolgreiche Implementierung der Liquid Biopsies wird von der Umsetzung multidisziplinärer Vorgehensweisen abhängen. Vor dem klinischen Routineeinsatz sind zurzeit noch zahlreiche prozedurale und Validierungsschritte notwendig, die von entsprechenden Konsortien wie Cancer-ID gemeinsam bearbeitet werden müssen. Die Untersuchung von Gewebsproben zur primären Diagnostik hinsichtlich einer exakten histopathologischen und molekularpathologischen Analyse liegt im Aufgabengebiet der Pathologie. Da im Rahmen von Liquid Biopsies umfangreiche Daten über Genome, sowohl somatische, tumorspezifische Veränderungen wie auch Keimbahnvarianten erhoben werden, kommt der Zusammenarbeit mit der Humangenetik bei 
der Umsetzung der Liquid Biopsy eine Schlüsselrolle zu. Die Humangenetik ist aufgrund ihrer Erfahrungen bei Sequenzierverfahren, Dateninterpretation, Datenbanken, Befunderstellung, Befundvermittlung von komplexen Datensätzen und Umgang mit familiären Situationen prädestiniert, sich in den Bereich der Liquid Biopsies einzubringen.

Da die Liquid Biopsies nicht von einem Fachbereich allein für sich reklamiert werden können, hängt die erfolgreiche und notwendige Beteiligung aller Fachrichtungen, insbesondere der Humangenetik und der Pathologie, ganz kritisch davon ab, inwieweit die vorgenannte Infrastruktur an den jeweiligen Standorten aufgebaut werden kann. Unsere Patienten werden am meisten von diesen neuen, vielversprechenden Ansätzen profitieren, wenn multidisziplinär zusammengearbeitet wird, was eine unabdingbare Voraussetzung für eine erfolgreiche Umsetzung sein wird.

\section{Korrespondenzadresse}

Univ.-Prof. Dr. M. R. Speicher

Institut für Humangenetik, Medizinische Universität Graz

Harrachgasse 21/8, 8010 Graz, Österreich michael.speicher@medunigraz.at

Danksagung. Die Autoren bedanken sich bei Frau Assoz. Prof. Mag. Dr. rer. nat. Ellen Heitzer und Herrn Assoz. Prof. Priv.-Doz. Dr. med. Jochen B. Geigl für kritische Durchsicht und Kommentierung des Manuskriptes.

Open access funding provided by Medical University of Graz.

\section{Einhaltung ethischer Richtlinien}

Interessenkonflikt. M.R. Speicher, A. Gerger und G. Hoefler geben an, dass kein Interessenkonflikt besteht.

Dieser Beitrag beinhaltet keine von den Autoren durchgeführten Studien an Menschen oder Tieren.

Open Access. Dieser Artikel wird unter der Creative Commons Namensnennung 4.0 International Lizenz (http://creativecommons.org/licenses/by/4.0/deed. de) veröffentlicht, welche die Nutzung, Vervielfältigung, Bearbeitung, Verbreitung und Wiedergabe in jeglichem Medium und Format erlaubt, sofern Sie den/die ursprünglichen Autor(en) und die Quelle ordnungsgemäß nennen, einen Linkzur Creative Commons Lizenz beifügen und angeben, ob Änderungen vorgenommen wurden.

\section{Literatur}

1. Stratton MR, Campbell PJ, Futreal PA (2009) The cancer genome. Nature 458(7239):719-724

2. Vogelstein B, Papadopoulos N, Velculescu VE, Zhou S, Diaz LA Jr., Kinzler KW (2013) Cancer genome landscapes. Science 339(6127):1546-1558

3. Speicher MR, Pantel K (2014) Tumor signatures in the blood. Nat Biotechnol 32(5):441-443

4. Pantel K, BrakenhoffRH, Brandt B (2008) Detection, clinical relevance and specific biological properties of disseminating tumour cells. Nat Rev Cancer 8(5):329-340

5. Schwarzenbach H, Hoon DS, Pantel K (2011) Cellfree nucleic acids as biomarkers in cancer patients. Nat Rev Cancer 11(6):426-437

6. Im H, Shao H, Park Yl, Peterson VM, Castro CM, Weissleder R et al (2014) Label-free detection and molecular profiling of exosomes with a nanoplasmonic sensor. Nat Biotechnol 32(5):490-495

7. Heitzer E, Auer M, UlzP, Geigl JB, Speicher MR (2013) Circulating tumor cells and DNA as liquid biopsies. Genome Med 5(8):73

8. Crowley E, Di Nicolantonio F, Loupakis F, Bardelli A (2013) Liquid biopsy: monitoring cancer-genetics in the blood. Nat Rev Clin Oncol 10(8):472-484

9. Diaz LA Jr., Bardelli A (2014) Liquid biopsies: genotyping circulating tumor DNA. J Clin Oncol 32(6):579-586

10. Heitzer E, Ulz P, Geigl JB (2015) Circulating tumor DNA as a liquid biopsy for cancer. Clin Chem 61(1):112-123

11. Alix-PanabieresC,PantelK(2016)Clinical applications of circulating tumor cells and circulating tumor DNA as liquid biopsy. Cancer Discov 6(5):479-491

12. Nass JB, Phillips J, Patlak M (2015) Policy issues in the development and adoption of biomarkers for molecularly targeted cancer therapies. The National Academies Press, Washington

13. ActionableGenome Consortium (2014) Actionable Genome Consortium to guide NGS in cancer. Nat Biotech 32(10):965

14. Roychowdhury S, lyer MK, Robinson DR, Lonigro RJ, Wu YM, CaoXetal (2011) Personalized oncology through integrative high-throughput sequencing: a pilot study. Sci TransI Med 3(111):111 ra21

15. Ulz P, Belic J, Graf R, Auer M, Lafer I, Fischereder $K$ et al (2016) Whole-genome plasma sequencing reveals focal amplifications as a driving force in metastatic prostate cancer. Nat Commun 7:12008

16. Roychowdhury S, Chinnaiyan AM (2016) Translating cancer genomes and transcriptomes for precision oncology. CA Cancer J Clin 66(1):75-88

\section{Kurzinformationen für \\ Patienten mit Seltenen \\ Erkrankungen}

Kooperationsprojekt des Ärztlichen Zentrums für Qualität in der Medizin (ÄZQ) und der ACHSE

Gemeinsam erstellen das ÄZQ und die ACHSE Kurzinformationen für Patienten zu zehn ausgewählten Seltenen Erkrankungen.

Folgende Kurzinformationen stehen allen niedergelassenen Ärzten zum Ausdrucken kostenlos zur Verfügung, um sie den Patienten mitzugeben:

- Adrenoleukodystrophie

- Deletionssyndrom 22Q11

- Doose-Syndrom

- Marfan-Syndrom

- Morbus Ormond

- Morbus Osler

- Mukoviszidose

- Erblich bedingte Netzhauterkrankungen

- Sarkoidose

Sie finden diese auf der gemeinsamen Website der Bundesärztekammer und der Kassenärztlichen Bundesvereinigung: http://www.patienten-information.de/ kurzinformationen/seltene-erkrankungen.

Zu Seltenen Erkrankungen gibt es oft keine oder nur schwer verständliche Informationen, weil sie in medizinischer Fachsprache oder in einer fremden Sprache verfasst sind. Im Bereich der Seltenen Erkrankungen besteht deshalb ein großer Bedarf an patientenorientierten Krankheitsbeschreibungen.

Das ÄZQ und die ACHSE haben sich deshalb zusammengetan und zehn Seltene Erkrankungen ausgewählt, zu denen nach einheitlichen Kriterien Kurzinformationen erarbeitet werden. Hierbei gilt es, nicht nur die Krankheitsanzeichen, sondern auch Untersuchungs- und Behandlungsmöglichkeiten sowie praktische Tipps zum besseren Umgang mit der Erkrankung aufzunehmen.

Quelle: Ärztliches Zentrum für Qualität in der Medizin (ÄZQ), www.aezq.de 